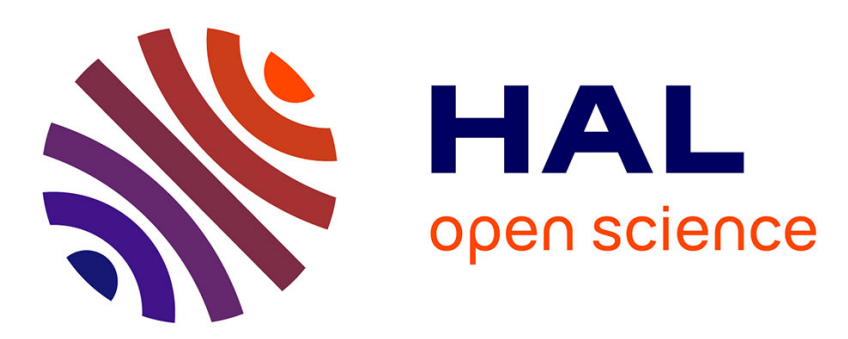

\title{
Nonlinear travelling waves in rotating Hagen-Poiseuille flow
}

Benoît Pier, Rama Govindarajan

\section{To cite this version:}

Benoît Pier, Rama Govindarajan. Nonlinear travelling waves in rotating Hagen-Poiseuille flow. Fluid Dynamics Research, 2018, 10.1088/1873-7005/aaaa9f . hal-01693277

\section{HAL Id: hal-01693277 https://hal.science/hal-01693277}

Submitted on 26 Jan 2018

HAL is a multi-disciplinary open access archive for the deposit and dissemination of scientific research documents, whether they are published or not. The documents may come from teaching and research institutions in France or abroad, or from public or private research centers.
L'archive ouverte pluridisciplinaire HAL, est destinée au dépôt et à la diffusion de documents scientifiques de niveau recherche, publiés ou non, émanant des établissements d'enseignement et de recherche français ou étrangers, des laboratoires publics ou privés. 


\title{
Nonlinear travelling waves in rotating Hagen-Poiseuille flow
}

\author{
Benoît PIER ${ }^{1}$ and Rama GOVINDARAJAN ${ }^{2}$ \\ ${ }^{1}$ Laboratoire de mécanique des fluides et d'acoustique, \\ CNRS-École centrale de Lyon-Université de Lyon 1-INSA de Lyon, \\ 36 avenue Guy-de-Collongue, 69134 Écully, France. \\ ${ }^{2}$ International Centre for Theoretical Sciences, \\ Tata Institute of Fundamental Research, \\ Survey No. 151, Shivakote, Hesaraghatta Hobli, Bengaluru 560 089, India.
}

\begin{abstract}
The dynamics of viscous flow through a rotating pipe is considered. Small-amplitude stability characteristics are obtained by linearizing the NavierStokes equations around the base flow and solving the resulting eigenvalue problems. For linearly unstable configurations, the dynamics leads to fully developed finiteamplitude perturbations that are computed by direct numerical simulations of the complete Navier-Stokes equations. By systematically investigating all linearly unstable combinations of streamwise wave number $k$ and and azimuthal mode number $m$, for streamwise Reynolds numbers $\operatorname{Re}_{z} \leq 500$ and rotational Reynolds numbers $\operatorname{Re}_{\Omega} \leq 500$, the complete range of nonlinear travelling waves is obtained and the associated flow fields are characterized.
\end{abstract}




\section{Introduction}

The axial flow through a rotating pipe has been studied over decades, both for its fundamental importance and for its wide application in turbomachinery and other fluid machines. Rotation changes the basic nature of the instability of the laminar HagenPoiseuille flow $[1,2,3,4,5,6,7]$ and the subsequent route towards turbulence. The final turbulent state too is rather different from that in the non-rotating pipe [8].

The laminar flow through a non-rotating pipe is linearly stable at all Reynolds numbers in that all eigenmodes are exponentially decaying. The transition to turbulence takes place by means of a subcritical route, due to the non-normality of the stability operator, and the consequent algebraic growth of disturbances. In contrast, when the pipe wall is in rotation about the pipe axis, exponentially growing disturbances are supported, and the nonlinear evolution is supercritical. Pedley [1] showed in 1968 that the inviscid flow in the limit of strong pipe rotation and weak axial flow is linearly unstable to long wave disturbances. He extended his study to viscous flow in the following year [2] to show that rotating Hagen-Poiseuille flow (RHPF) in the same strong rotation limit is linearly unstable to long waves above a critical Reynolds number of 82.9. The mode that was found to be least stable was a corkscrew mode which propagates axially in the direction of the flow but rotates in a sense opposite to that of the walls of the pipe. Given the symmetries in the problem, this mode is equivalent to one which propagates axially in a sense opposite to the axial mean flow but rotates in the same sense as the pipe. Mackrodt [3] extended this study to lower rotation rates and showed that the flow can still be linearly unstable at fairly low rotation rates. This work also showed experimentally that residual swirl in the entry region can trigger instabilities. A comprehensive study of the linear stability of the RHPF was carried out by Cotton and Salwen [4], extending the earlier results to higher axial wavenumbers and a range of azimuthal mode numbers. By deriving neutral stability curves, they noticed that the critical Reynolds number is only weakly dependent on the rotation number provided that the latter is large enough, and that similarly the critical rotation number is only weakly dependent on the Reynolds number provided that the Reynolds number is large enough. In other words, the unstable region is approximately delimited by lines of either constant Reynolds number or constant rotation number. An asymptotic theory for the draining bathtub vortex was given by Foster [9], and there are a host of such studies of swirling flows where the pipe walls are stationary, which we do not discuss here. Analogously, Rusak and Wang [6] conducted an inviscid study of solid body rotation accompanied by uniform axial flow and found a critical swirl ratio, above which there are two kinds of nonlinear states.

Given that a Hagen-Poiseuille flow through a stationary pipe is linearly stable to all convective modes, this flow can obviously never be absolutely unstable. In contrast, RHPF can display modes of absolute instability, as was shown by Fernandez et al. [5]. These modes of absolute instability were obtained experimentally in the recent study of Miranda-Barea et al. [7]. They applied a spin-down to the pipe and could thus obtain 
the critical rotation rate for the switch from absolute instabilities to convective.

Some studies of the nonlinear evolution of disturbances have been carried out on the RHPF. Toplosky and Akylas [10] resolved a controversy as to whether the nonlinear evolution is supercritical or subcritical, and found no subcritical nonlinearities. All subsequent nonlinear studies to our knowledge, and also the present study, find only supercritical growth of perturbations in the nonlinear regime. In fact the boundary of linear neutral stability also marks the onset of nonlinear evolution of wave trains. This was seen explicitly in the study of Yang and Leibovich [11] which focused on the close vicinity of the neutral stability boundary. The predicted linear and nonlinear modes were confirmed qualitatively in the experiments of Imao et al. [12]. Furthermore, these experiments have shown how the initially sinusoidal perturbations adopted a sawtooth form in their nonlinear state. Far downstream, perturbations other than the least stable linear mode were triggered, and the disturbance spectrum became broadband. Landman [13] imposed the constraint of helical symmetry, and demonstrated that the nonlinear state goes through several bifurcations and results in a time-dependent flow. Barnes and Kerswell [14] found three-dimensional travelling waves which appeared after a bifurcation from the solutions found by Toplosky and Akylas [10].

A range of studies focus on pipes where the flow is not streamwise independent. One situation is of course when the pipe is finite, so entry and exit flows dictate the dynamics, see e.g. [15]. Dennis et al. [16] created a vortex breakdown in a pipe where one part was rotating faster than a critical rate. The fully turbulent flow through a rotating pipe is not a topic of the present study, but it is of interest to note that while rotation acts to destabilise the laminar flow and provide a supercritical route to turbulence, the resulting turbulence may be suppressed by rotation [8].

The objective of the present investigation is to systematically derive and characterize the family of nonlinear travelling-wave solutions sustained in rotating Hagen-Poiseuille flow. Therefore we study the nonlinear evolution of a class of travelling waves over a range of the relevant parameters in a periodic box. We show that the dynamics saturates into a nonlinear state of a system of travelling waves. In all cases, the most energetic nonlinear state has longer wavelength than that of the corresponding fastest growing linear eigenmode.

\section{Problem formulation}

In this study, the fluid flow is described by a vector velocity field $\mathbf{u}(\mathbf{x}, t)$ and a scalar pressure field $p(\mathbf{x}, t)$ that depend on position $\mathbf{x}$ and time $t$ and are governed by the incompressible Navier-Stokes equations

$$
\begin{aligned}
\frac{\partial \mathbf{u}}{\partial t}+(\mathbf{u} \cdot \nabla) \mathbf{u} & =\nu \Delta \mathbf{u}-\nabla p, \\
\nabla \cdot \mathbf{u} & =0,
\end{aligned}
$$

where $\nu$ is the kinematic viscosity of the fluid, and the pressure has been redefined to eliminate the constant fluid density from the equations. The flow occurs inside a pipe 
of radius $R$ which is rotating at rate $\Omega$ about its axis and no-slip boundary conditions prevail along the pipe walls. A fixed cylindrical coordinate system will be used where $r$, $\theta$ and $z$ denote radial, azimuthal and axial coordinates respectively, and $r=0$ coincides with the pipe axis.

In the RHPF configuration, the flow is driven by the combination of two factors: a constant axial pressure gradient and the rotating pipe walls. The resulting steady, axisymmetric and axially invariant base flow is then an exact superposition of an axial parabolic velocity profile $U_{z}$ and an azimuthal solid-body rotation $U_{\theta}$ depending only on the radial coordinate $r$ as

$$
U_{z}(r)=2 \bar{U}\left(1-\frac{r^{2}}{R^{2}}\right) \quad \text { and } \quad U_{\theta}(r)=\Omega r,
$$

where $\bar{U}$ is the mean axial velocity, associated with an axial pressure gradient of magnitude $G_{z}=8 \nu \bar{U} / R^{2}$. This flow is governed by two non-dimensional control parameters, the streamwise and azimuthal (or rotational) Reynolds numbers defined as

$$
\operatorname{Re}_{z} \equiv 2 \bar{U} R / \nu \quad \text { and } \quad \operatorname{Re}_{\Omega} \equiv 2 \Omega R^{2} / \nu
$$

respectively. With this definition, the ratio $\operatorname{Re}_{\Omega} / \operatorname{Re}_{z}$ corresponds to the ratio of the azimuthal pipe wall velocity to the mean axial flow velocity. In the sequel, units used for giving numerical results are based on the pipe diameter $2 R$ and the mean axial velocity $\bar{U}$.

Throughout this investigation, the total instantaneous flow fields are separated into basic and perturbation quantities as

$$
\begin{aligned}
& u_{r}^{t o t}(r, \theta, z, t)=u_{r}(r, \theta, z, t), \\
& u_{\theta}^{t o t}(r, \theta, z, t)=U_{\theta}(r)+u_{\theta}(r, \theta, z, t), \\
& u_{z}^{\text {tot }}(r, \theta, z, t)=U_{z}(r)+u_{z}(r, \theta, z, t), \\
& p^{t o t}(r, \theta, z, t)=-G_{z} z+p(r, \theta, z, t),
\end{aligned}
$$

whether the perturbation is of small amplitude or not. Replacing these expansions into the Navier-Stokes equations $(1,2)$ then yields the governing equations for the perturbation flow fields $u_{r}, u_{\theta}, u_{z}$ and $p$.

When analysing the dynamics of small-amplitude perturbations, the disturbances may be written in normal-mode form as

$$
\begin{aligned}
& u_{r}(r, \theta, z, t)=\hat{u}_{r}(r) \operatorname{expi}(k z+m \theta-\omega t), \\
& u_{\theta}(r, \theta, z, t)=\hat{u}_{\theta}(r) \exp \mathrm{i}(k z+m \theta-\omega t), \\
& u_{z}(r, \theta, z, t)=\hat{u}_{z}(r) \operatorname{expi}(k z+m \theta-\omega t), \\
& p(r, \theta, z, t)=\hat{p}(r) \operatorname{expi}(k z+m \theta-\omega t),
\end{aligned}
$$

where $k$ is an axial wave number, $m$ an azimuthal mode number and $\omega$ a (complex) frequency. Linearization of the governing equations then leads to eigenvalue problems, the solution of which yields the $\omega$-eigenvalue spectrum together with the associated 
eigenfunctions $\hat{u}_{r}(r), \hat{u}_{\theta}(r), \hat{u}_{z}(r)$ and $\hat{p}(r)$, for each setting of $k, m, \operatorname{Re}_{z}$ and $\operatorname{Re}_{\Omega}$. Usually, the spectrum is dominated by a single eigenvalue and its identification then yields the linear dispersion relation as

$$
\omega=\omega^{\operatorname{lin}}\left(k, m ; \operatorname{Re}_{z}, \operatorname{Re}_{\Omega}\right) .
$$

In unstable situations, characterized by positive growth rates $\omega_{i} \equiv \operatorname{Im} \omega$, an initial spatially periodic small-amplitude perturbation may be exponentially amplified in time and eventually reach finite amplitudes, thus be governed by the full nonlinear equations. The resulting nonlinear dynamics is then investigated by using spatial Fourier series of the form

$$
\begin{aligned}
& u_{r}(r, \theta, z, t)=\sum_{n} u_{r}^{(n)}(r, t) \exp \mathrm{i} n(k z+m \theta), \\
& u_{\theta}(r, \theta, z, t)=\sum_{n} u_{\theta}^{(n)}(r, t) \exp \mathrm{i} n(k z+m \theta), \\
& u_{z}(r, \theta, z, t)=\sum_{n} u_{z}^{(n)}(r, t) \exp \mathrm{i} n(k z+m \theta), \\
& p(r, \theta, z, t)=\sum_{n} p^{(n)}(r, t) \exp \mathrm{i} n(k z+m \theta),
\end{aligned}
$$

and integrating in time the nonlinear temporal evolution problem consisting in a system of coupled partial differential equations for the components $u_{r}^{(n)}(r, t), u_{\theta}^{(n)}(r, t), u_{z}^{(n)}(r, t)$ and $p^{(n)}(r, t)$. This is equivalent to considering a finite domain with periodic boundary conditions and investigating the dynamics of finite-amplitude disturbances at prescribed total axial pressure gradient. The numerical implementation of this time-marching problem uses a second-order accurate predictor-corrector fractional-step method, similar to the one described in detail in [17]. Complete expressions and derivations of the linear and nonlinear governing equations as well as numerical solution methods may be found in $[18]$.

It should be emphasized that the use of single spatial Fourier series in expansions (14-17), instead of double series using terms of the form expi $(n k z+\operatorname{lm} \theta)$ with $n \neq l$, is motivated by our objective of computing primary nonlinear travelling wave solutions, rather than studying their stability with respect to secondary perturbations. Indeed, starting from a linearly unstable small-amplitude initial perturbation of the form expi $(k z+m \theta)$, the nonlinearities of the Navier-Stokes equations only promote spatial harmonics of the form exp in $(k z+m \theta)$, while terms of the form expi $(n k z+\ln \theta)$ with $n \neq l$ would only be generated by secondary instabilities.

\section{Results}

\subsection{Linear dynamics}

The dynamics of small-amplitude perturbations is governed by the dispersion relation (13) derived from a normal-mode analysis of the Navier-Stokes equations linearized around the base flow (3). In this subsection we repeat some of the results 
obtained by previous authors to validate our approach and identify the parameter space of interest for the subsequent investigation of nonlinear travelling-wave solutions.

Temporal growth rates $\omega_{i}$ corresponding to the most unstable mode are shown in figure 1 over the $\left(\operatorname{Re}_{z}, k\right)$-plane for $\operatorname{Re}_{z} \leq 500$ and different values of $\operatorname{Re}_{\Omega}$ and $m$. For $m=-1$ (figure $1 \mathrm{a}-\mathrm{d}$ ), the RHPF becomes already unstable at $\operatorname{Re}_{\Omega}=60$, while higher values of $\operatorname{Re}_{\Omega}$ are required for $m=-2$ (figure $1 \mathrm{e}-\mathrm{f}$ ) and $m=-3$ (figure $1 \mathrm{~g}-\mathrm{h}$ ). These results fully agree with those obtained by Cotton and Salwen [4].

The critical curves for onset of instability in the $\left(\operatorname{Re}_{z}, \mathrm{Re}_{\Omega}\right)$-plane are shown in figure 2 for $m=-1,-2$ and -3 . As already noticed by [4], these critical curves asymptote towards constant values of $\mathrm{Re}_{z}$ or of $\mathrm{Re}_{\Omega}$. Except for low values of both $\mathrm{Re}_{z}$ and $\mathrm{Re}_{\Omega}$, onset of instability thus only depends on the lowest of these two control parameters. For parameter values within the linearly unstable regions, growing perturbations lead to a fully developed regime, analysed in detail in the rest of this paper.

\subsection{Nonlinear travelling waves}

In configurations where the base flow displays linear temporal instability, a smallamplitude perturbation with a positive growth rate is exponentially amplified and eventually reaches finite-amplitude levels. The nonlinear terms in the Navier-Stokes equations can then no longer be neglected and the dynamics is governed by the complete nonlinear equations. The aim of this investigation is to systematically derive and characterize the resulting fully developed travelling wave solutions for $\operatorname{Re}_{z} \leq 500$ and $\operatorname{Re}_{\Omega} \leq 500$.

The approach is here based on temporal evolution problems investigated by direct numerical simulations of the Navier-Stokes equations. Starting from a small-amplitude initial perturbation, the evolution is first dictated by linear dynamics before nonlinear effects come into play. In the absence of secondary instabilities, a fully developed regime is reached with spatial periodicity imposed by the prescribed values of streamwise wave number $k$ and azimuthal mode number $m$. As already mentioned in the Introduction, no sign of a subcritical transition has been found. This approach is therefore deemed to yield all nonlinear wave solutions that may prevail in the RHPF, for the controlparameter region under consideration.

A typical scenario may be illustrated by considering the temporal evolution of a perturbation at $k=1$ and $m=-1$ for $\operatorname{Re}_{z}=250$ and $\operatorname{Re}_{\Omega}=200$. At these parameter values the linear dispersion relation yields a complex frequency of $\omega^{\text {lin }}=-0.42+0.44 \mathrm{i}$, with a positive temporal growth rate. Figure 3 shows the temporal evolution of the energy $E^{(n)}$ contained in each spatial harmonic together with the total $E^{\mathrm{nl}} \equiv \sum_{n \geq 0} E^{(n)}$, both on logarithmic and linear scales; the energy $E^{(n)}$ is here defined as the spatially averaged kinetic energy per unit volume associated with the Fourier component corresponding to $n$ and $-n$ of the expansions (14-16).

In the first stage of the evolution, here approximately for $t<0.15$, the perturbation 

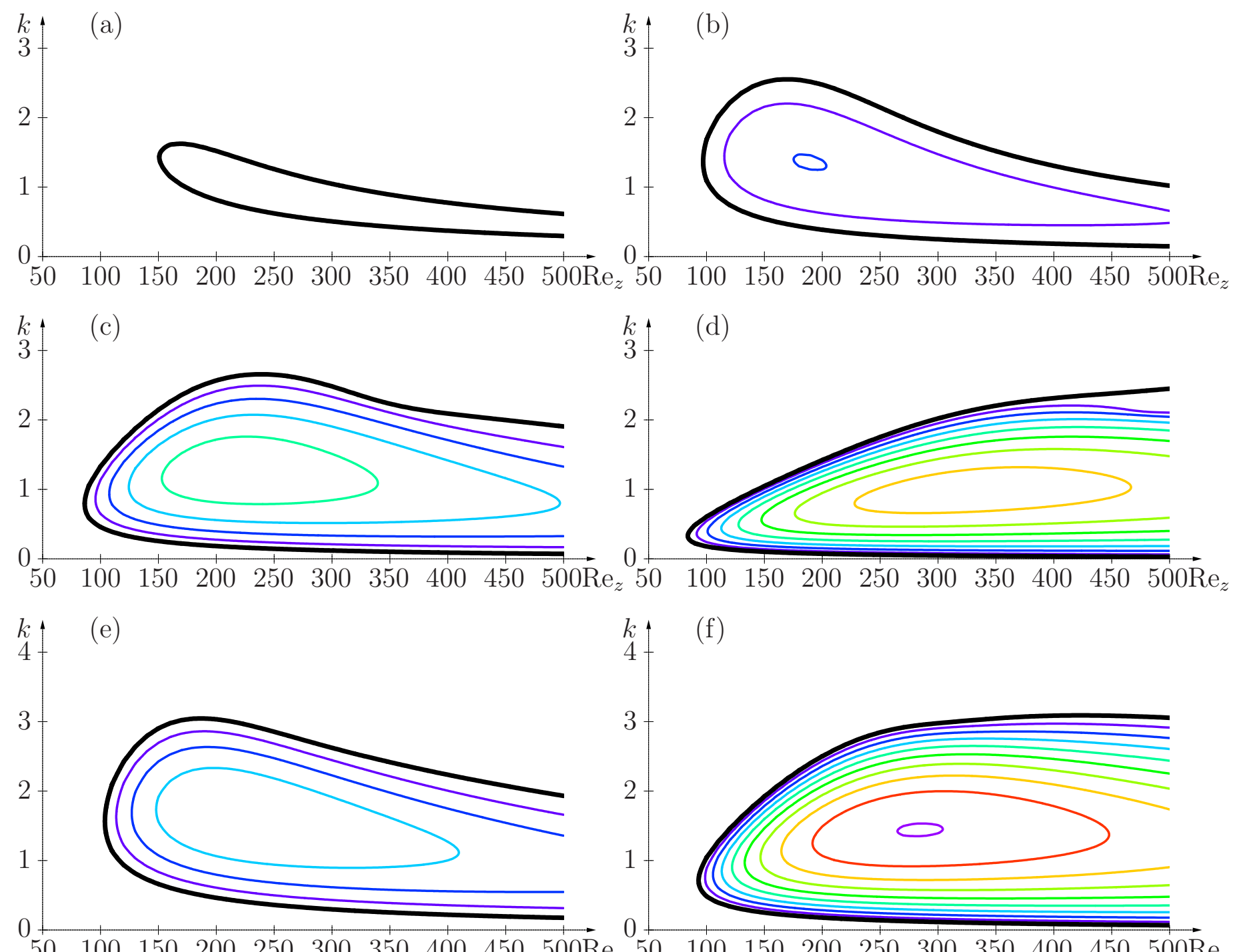

(f)

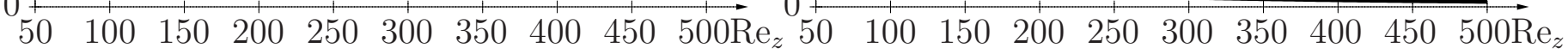
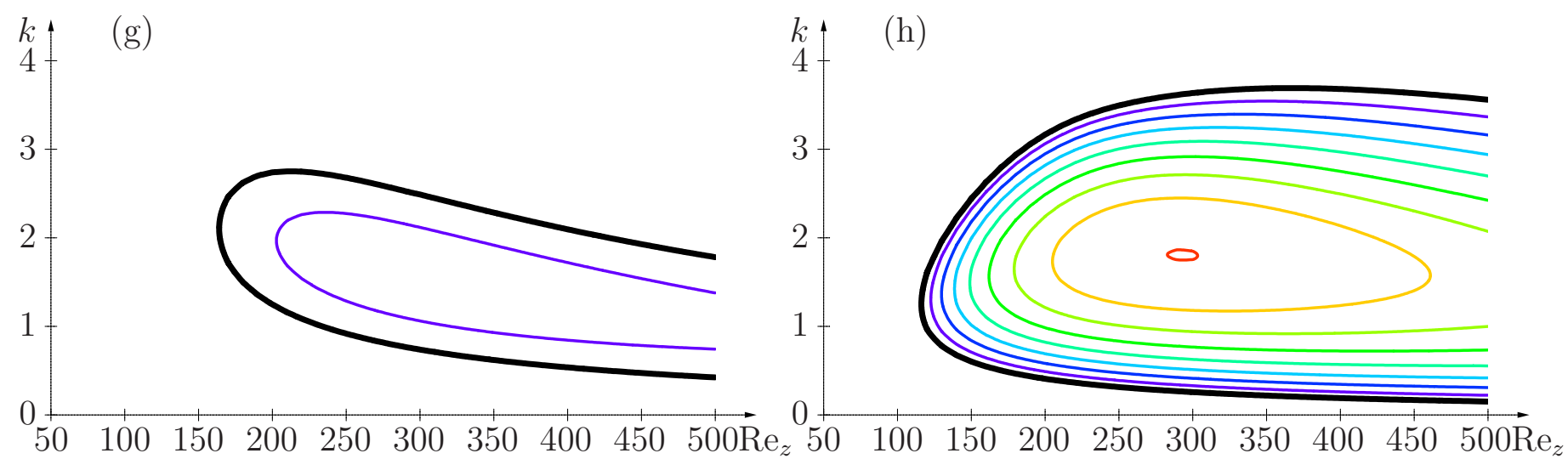

Figure 1. Temporal growth rate $\omega_{i}$ in $\left(\operatorname{Re}_{z}, k\right)$-plane for (a) $m=-1, \operatorname{Re}_{\Omega}=60$, (b) $m=-1, \operatorname{Re}_{\Omega}=100$, (c) $m=-1, \operatorname{Re}_{\Omega}=200$, (d) $m=-1, \operatorname{Re}_{\Omega}=500$, (e) $m=-2, \operatorname{Re}_{\Omega}=200$, (f) $m=-2, \operatorname{Re}_{\Omega}=500$, (g) $m=-3, \operatorname{Re}_{\Omega}=200$ and (h) $m=-3, \operatorname{Re}_{\Omega}=500$. Thick black lines correspond to the marginal curve $\omega_{i}=0$ and thin coloured lines to positive growth rates $\omega_{i}=0.1,0.2,0.3,0.4, \ldots$ 


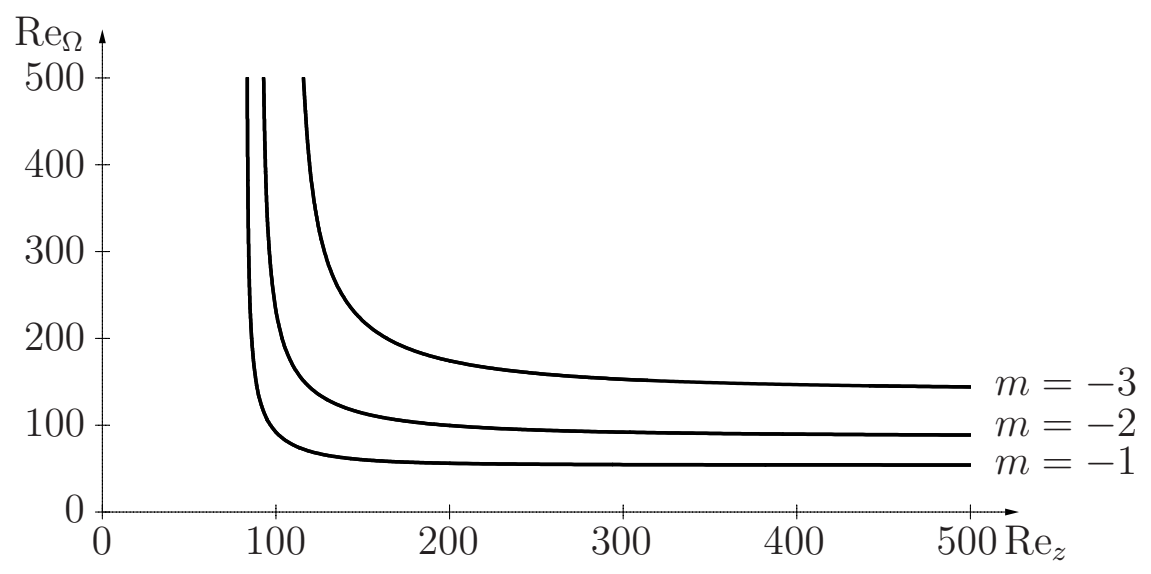

Figure 2. Neutral curves in $\left(\operatorname{Re}_{z}, \operatorname{Re}_{\Omega}\right)$-plane for $m=-1,-2,-3$.
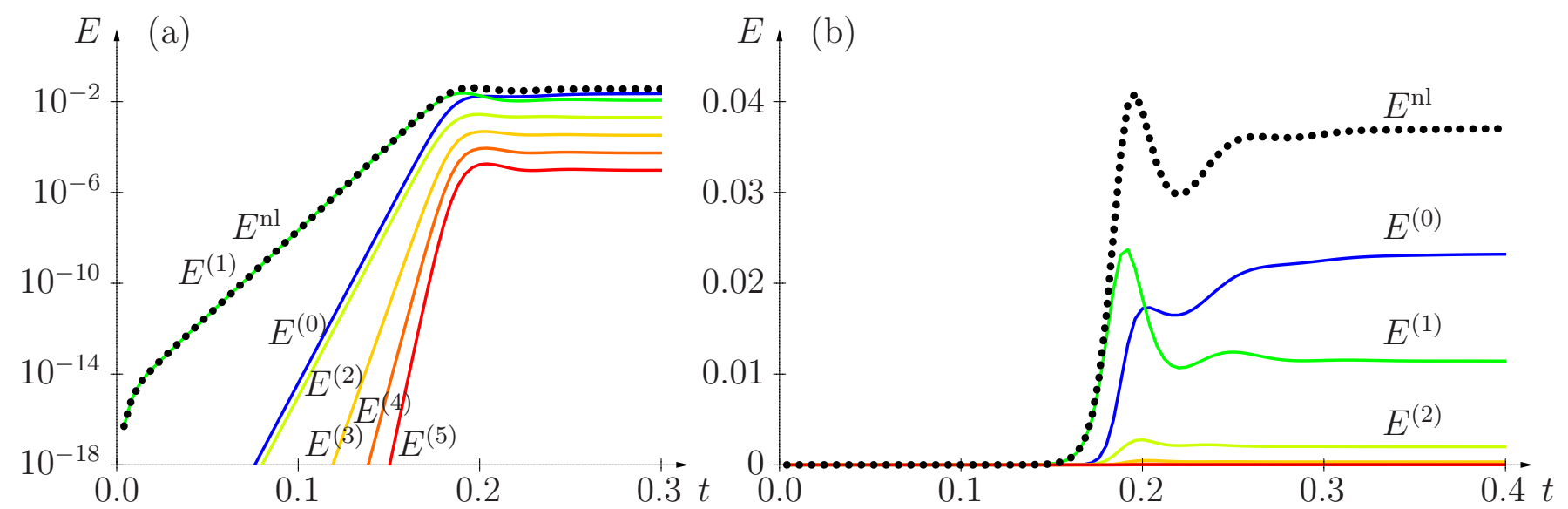

Figure 3. Temporal evolution of $E^{(n)}$, kinetic energy contained in each spatial Fourier component, and of total $E^{\mathrm{nl}}$, resulting from a small-amplitude initial perturbation at $k=1$ and $m=-1$ for $\operatorname{Re}_{z}=250$ and $\operatorname{Re}_{\Omega}=200$, plotted on (a) logarithmic and (b) linear scales.

is seen to grow exponentially: the energy $E^{(1)}(t)$ in the fundamental component is amplified as $\mathrm{e}^{2 \omega_{i} t}$, while the higher harmonics $(n \geq 2)$ are slaved by nonlinear interaction to the fundamental as $E^{(n)}(t) \propto\left[E^{(1)}(t)\right]^{n}$ and the mean-flow correction as $E^{(0)}(t) \propto\left[E^{(1)}(t)\right]^{2}$, see figure $3($ a). The nonlinearities are stabilizing and therefore lead to saturation at finite amplitude levels. In the absence of secondary instabilities, a fully nonlinear travelling-wave solution is then reached in the long-time limit with spatial periodicity imposed by the prescribed values of $k$ and $m$ and constant energy levels, see figure 3(b). For the final perturbation velocity and pressure fields (14-17) associated with this nonlinear travelling solution, each of the $n$th spatial Fourier components is found to depend on time as $\exp \left(-\mathrm{i} n \omega^{\mathrm{nl}} t\right)$ for some real frequency $\omega^{\mathrm{nl}}$. This means that the flow fields are $2 \pi$-periodic functions of $k z+m \theta-\omega^{\mathrm{nl}} t$, which is characteristic of threedimensional solutions that travel without deforming. The nonlinear frequency $\omega^{\mathrm{nl}}$ is related to the axial wave propagation speed as $\omega^{\mathrm{nl}} / k$ and to the azimuthal wave rotation rate as $\omega^{\mathrm{nl}} / m$. Its value is obtained by the numerical simulations, which therefore yield 


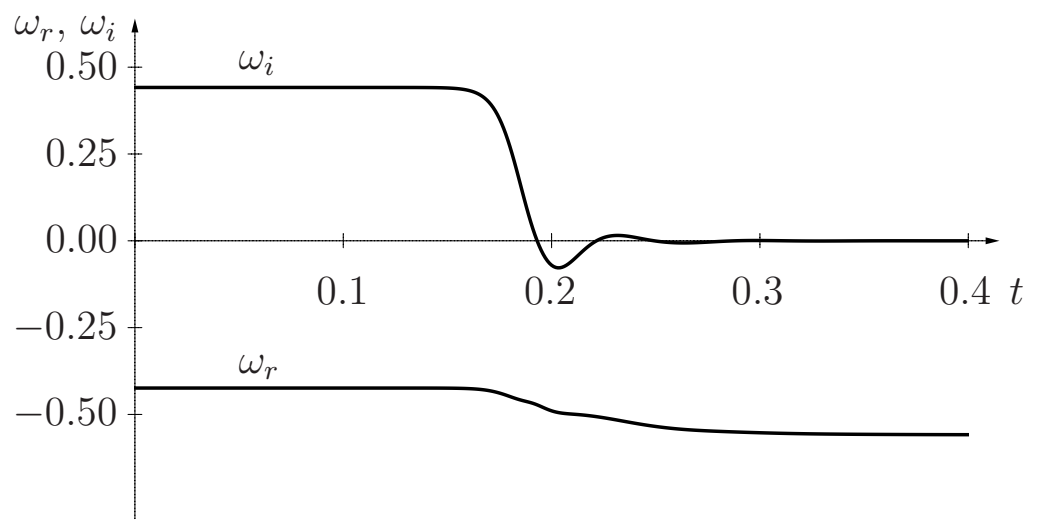

Figure 4. Temporal evolution of instantaneous frequency $\omega=\omega_{r}+\mathrm{i} \omega_{i}$ from the linear growth phase $\left(\omega^{\text {lin }}=-0.42+0.44 \mathrm{i}\right)$ to the saturation regime $\left(\omega^{\mathrm{nl}}=-0.56\right)$. Same configuration as figure 3 .

the nonlinear dispersion relation

$$
\omega^{\mathrm{nl}}\left(k, m ; \operatorname{Re}_{z}, \operatorname{Re}_{\Omega}\right) .
$$

In the example illustrated here, $\omega^{\mathrm{nl}}=-0.56$. Monitoring the temporal evolution of the instantaneous complex frequency, figure 4 shows how the growth rate varies from $\omega_{i}=0.44$ in the linear growth phase to a vanishing value in the saturation regime, while the real part of the frequency decreases from $\omega_{r}=-0.42$ to $\omega_{r}=-0.56$. As shown below (see figure 6a), this frequency reduction in the nonlinear régime is associated with the development of a negative mean-flow correction.

Note that the fully developed nonlinear travelling waves could have been obtained by an iterative Newton-Raphson search procedure, a technique that we have used and implemented in a similar context for the three-dimensional rotating-disk boundary layer [19]. For the RHPF, however, that was not necessary since for the parameter space under consideration the time-marching procedure outlined in $\S 2$ was always found to converge towards the desired finite-amplitude travelling wave solutions and, moreover, provides information about the transition from the linear growth phase to the saturated regime.

The velocity fields of this nonlinear travelling-wave solution are given in figure 5 . Isolines of the axial velocity are shown in figure 5(a) and (b) for the nonlinear perturbation and the total flow respectively. The velocity field in a cross-section of the pipe is shown in 5(c) and (d), again for the perturbation and total flow. Due to the travelling-wave structure of this solution, plots at a different axial position $z$ or different time $t$ only differ from those shown in figure 5 by rotation in $\theta$. The axial disturbance velocity component (figure 5a) is mostly negative and non-axisymmetric. Thus the resulting total axial velocity (figure 5b) is significantly reduced in comparison with the base Poiseuille profile and displays a maximum value slightly in excess of 1.6 off axis. Recall that the maximum axial Poiseuille velocity is 2 since the mean velocity $\bar{U}$ is used as unit. 

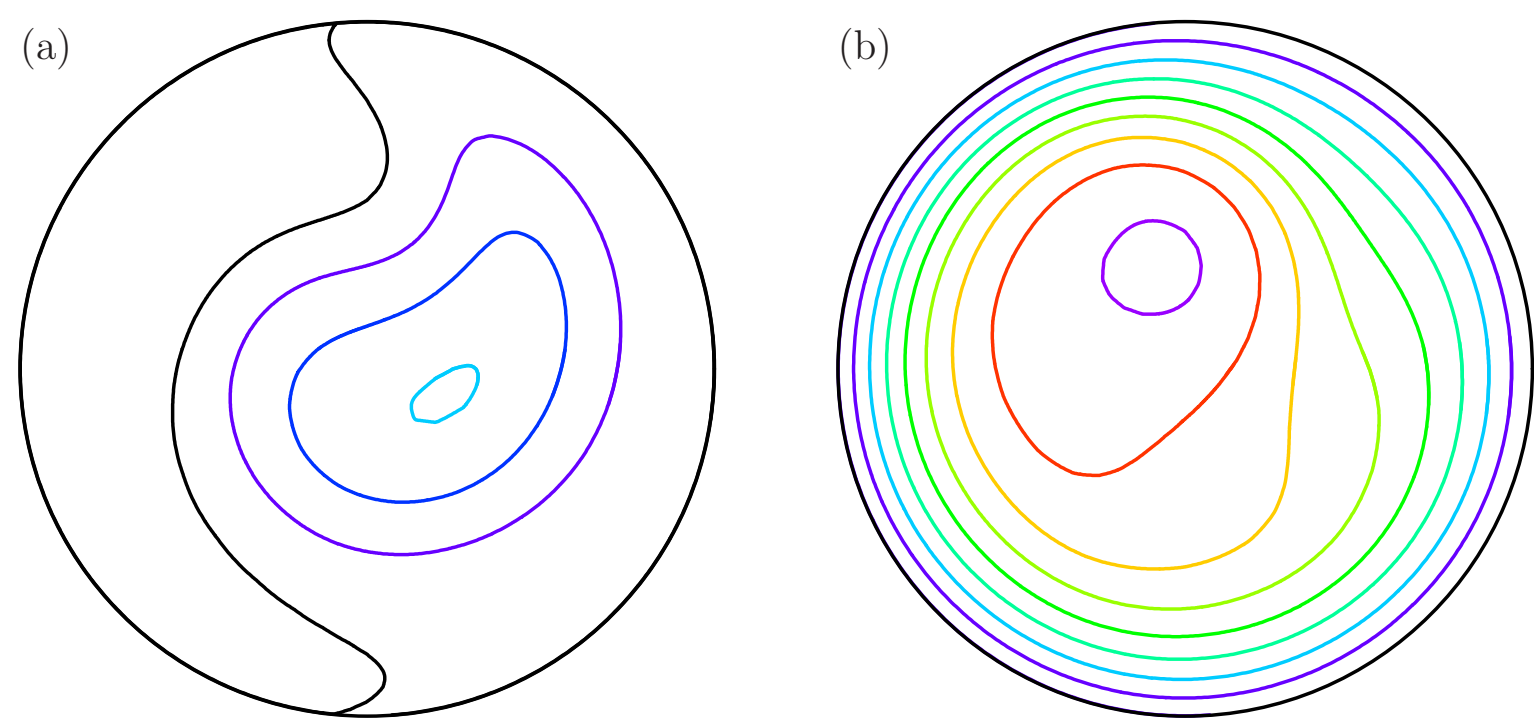

(c)

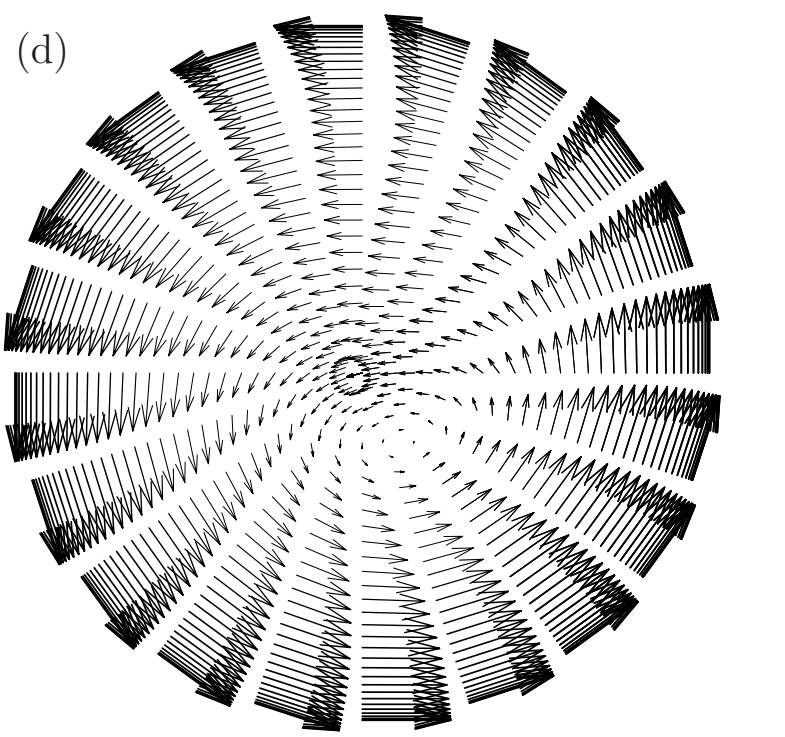

Figure 5. Velocity fields of saturated nonlinear travelling wave at $k=1$ and $m=-1$ for $\operatorname{Re}_{z}=250$ and $\operatorname{Re}_{\Omega}=200$ : (a,b) axial component and (c,d) in-plane velocity for $(\mathrm{a}, \mathrm{c})$ perturbation and $(\mathrm{b}, \mathrm{d})$ total flow. In $(\mathrm{a})$, coloured lines correspond to negative velocities $-0.2,-0.4$ and -0.6 . In (b), coloured lines correspond to positive velocities $0.2, \ldots, 1.6$. Black lines indicate vanishing values.

The development of nonlinearities produce higher harmonics as well as an axisymmetric mean flow correction associated with the non-periodic component of the perturbation. Figure 6 compares the mean profiles prevailing in the fully developed regime with those of the unperturbed base flow, both for the axial (figure 6a) and the azimuthal (figure 6b) components. The effect of this mean flow correction is to reduce the total axial flowrate $Q_{\text {tot }}$ as well as the total angular momentum $M_{\text {tot }}$ of the flow: here $Q_{\mathrm{tot}} / Q_{\mathrm{bf}}=0.88$ and $M_{\mathrm{tot}} / M_{\mathrm{bf}}=0.97$, where $Q_{\mathrm{bf}}$ and $M_{\mathrm{bf}}$ refer to flow rate and momentum prevailing for the unperturbed base flow.

By varying the disturbance wave numbers $k$ and $m$ and the base flow control 

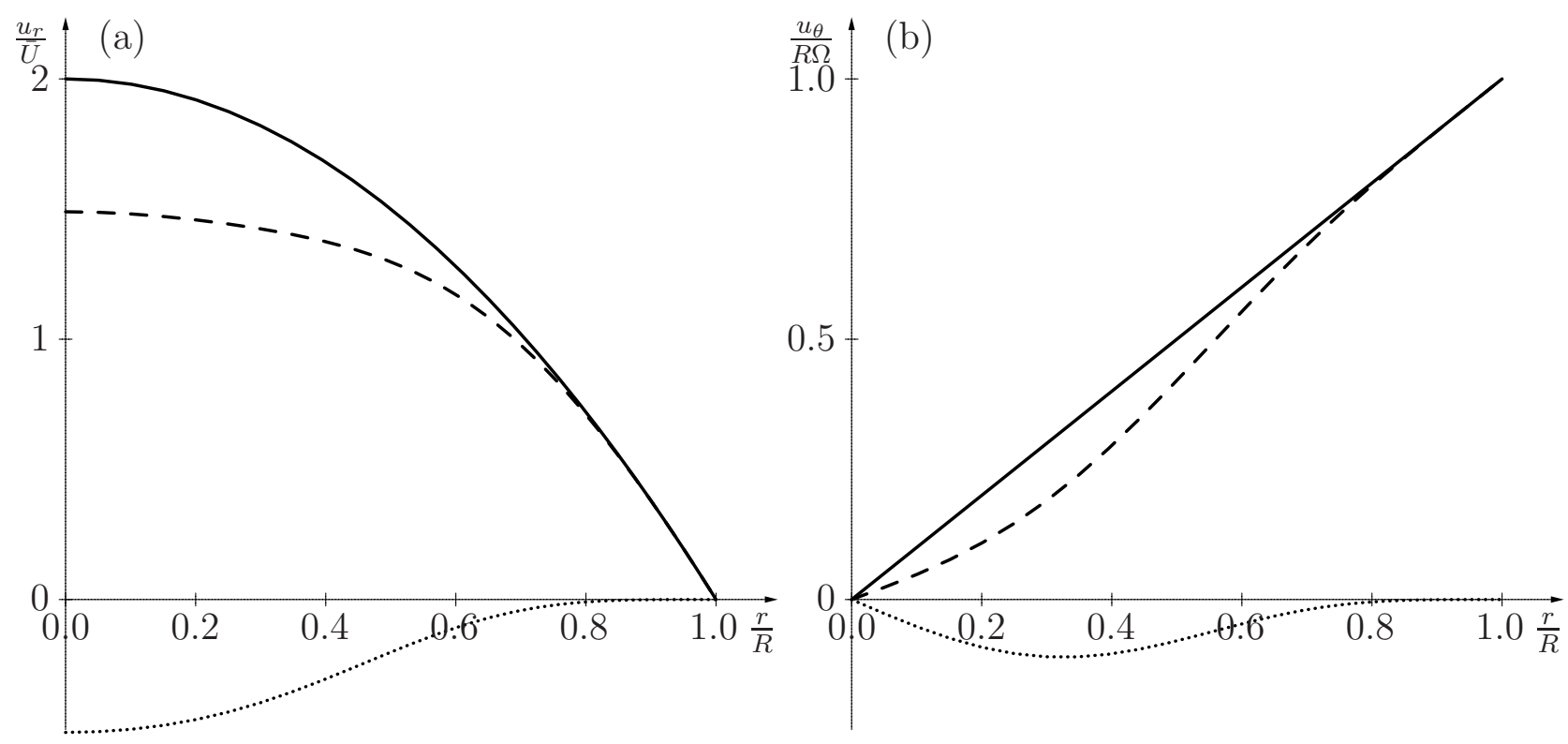

Figure 6. (a) Axial and (b) azimuthal mean velocity profiles prevailing for the fully developed nonlinear travelling wave: comparison of base profile (solid), mean flow correction (dotted) and total mean profile (dashed). Same parameter settings as in figures 3 and 5 .

parameters $\operatorname{Re}_{z}$ and $\mathrm{Re}_{\Omega}$, the complete set of nonlinear travelling waves may be systematically computed. In the present investigation we have covered the full range of nonlinear solutions for $\operatorname{Re}_{z} \leq 500$ and $\operatorname{Re}_{\Omega} \leq 500$.

Isolines of the total disturbance energy $E^{\mathrm{nl}}$ associated with the nonlinear travelling waves are plotted in figure 7 in the $\left(\operatorname{Re}_{z}, k\right)$-plane for selected values of $m$ and $\operatorname{Re}_{\Omega}$. For $m=-1$ and $\operatorname{Re}_{\Omega}=100$, comparison of the linear temporal growth rates shown in figure 1(b) with the energy levels of the nonlinear saturated travelling waves shown in figure $7(\mathrm{a})$ clearly demonstrates the supercritical nature of the nonlinearities: the amplitude of nonlinear travelling waves vanishes as the critical curve for instability is approached. However, the largest values of $E^{\mathrm{nl}}$ do not coincide with the strongest linear temporal growth rates. Indeed, for a given value of $\mathrm{Re}_{z}$, the strongest temporal growth rate occurs approximately in the center of the unstable $k$-range, while the largest nonlinear amplitudes are found towards lower values of $k$. Similar conclusions hold for all configurations investigated here, comparing figures $1(\mathrm{c})$ with $7(\mathrm{~b}), 1(\mathrm{e})$ with $7(\mathrm{c})$, 1(f) with $7(\mathrm{~d}), 1(\mathrm{~g})$ with $7(\mathrm{e})$ and $1(\mathrm{~h})$ with $7(\mathrm{f})$, respectively.

The mean axial flowrate corrections and angular momentum corrections due to the fully developed nonlinear travelling waves are shown in figures 8 and 9 , respectively. In these figures, mean-flow-correction quantities are shown relative to the values prevailing for the underlying base flows.

As expected by the supercritical nature of the instabilities, the flow-rate correction curves shown in figure 8 follow very similar trends to the energy curves of figure 7 . Comparison of panels corresponding to the same settings of $m$ and $\operatorname{Re}_{\Omega}$ reveals that the largest flow-rate corrections approximately correspond to the largest disturbance energy 

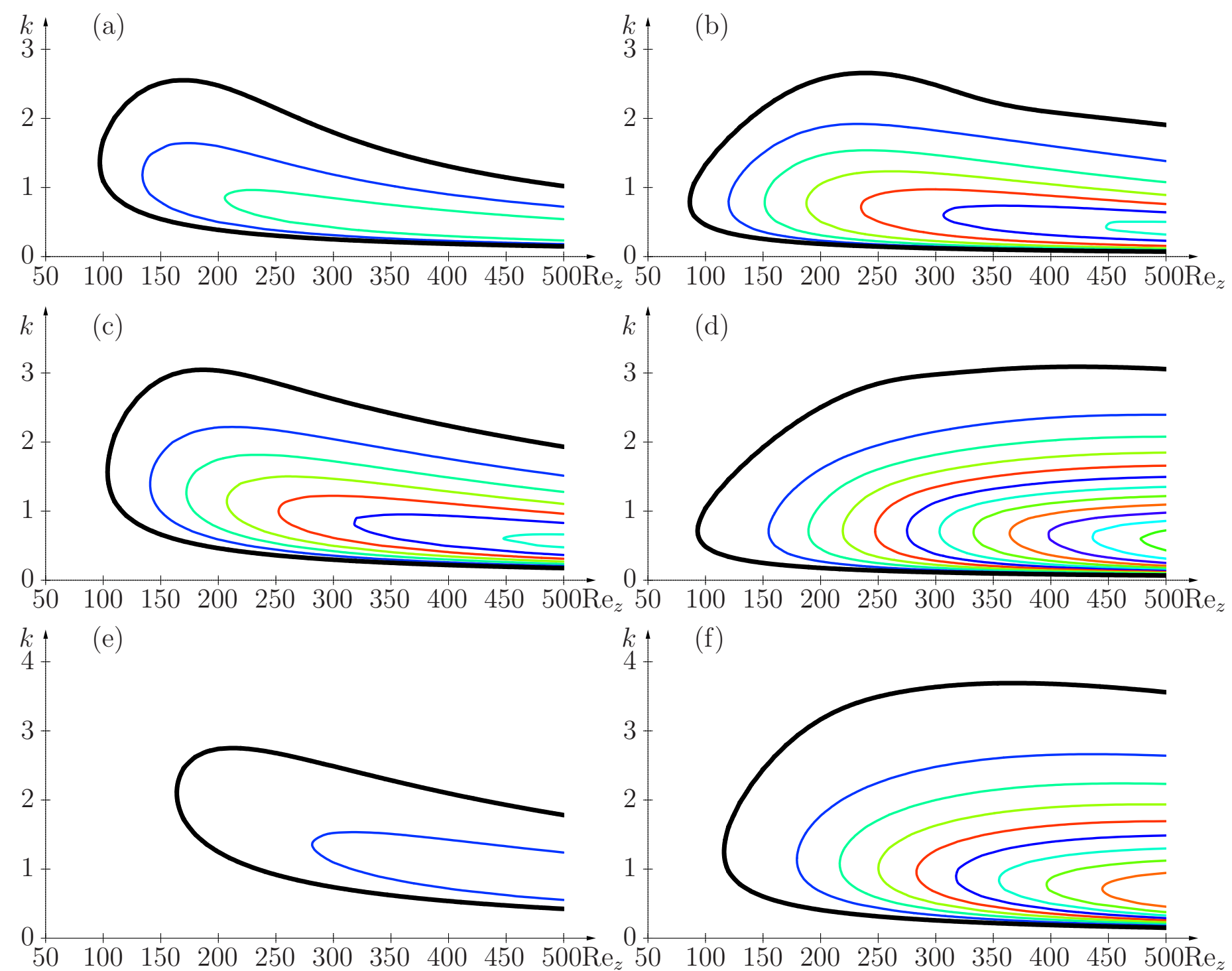

(f)

Figure 7. Levels of total disturbance energy $E^{n l}$ in the $\left(\operatorname{Re}_{z}, k\right)$-plane for (a) $m=-1$, $\operatorname{Re}_{\Omega}=100$, (b) $m=-1, \operatorname{Re}_{\Omega}=200$, (c) $m=-2, \operatorname{Re}_{\Omega}=200$, (d) $m=-2, \operatorname{Re}_{\Omega}=500$, (e) $m=-3, \operatorname{Re}_{\Omega}=200$, (f) $m=-3, \operatorname{Re}_{\Omega}=500$. Thick black lines correspond to the marginal curve and thin coloured lines to levels $E^{\mathrm{nl}}=0.005,0.010,0.015, \ldots$

levels.

Monitoring the mean angular momentum corrections as the control parameters are varied a slightly different pattern emerges (figure 9): while the values obviously still decay towards the critical curves, it is found that largest contributions prevail for axial wavenumbers in the center of the unstable $k$-range. These computations thus reveal that the strength of axial and azimuthal mean-flow corrections significantly depend on the wavenumber $k$ and that the axial corrections are more important for long wavelength perturbations. Thus the evolution of the relative angular momentum shown in figure 9 more closely follows that of the linear temporal growth rate (figure 1) than that of the saturated energy levels (figure 7). Unlike the linear temporal growth rates that display a 

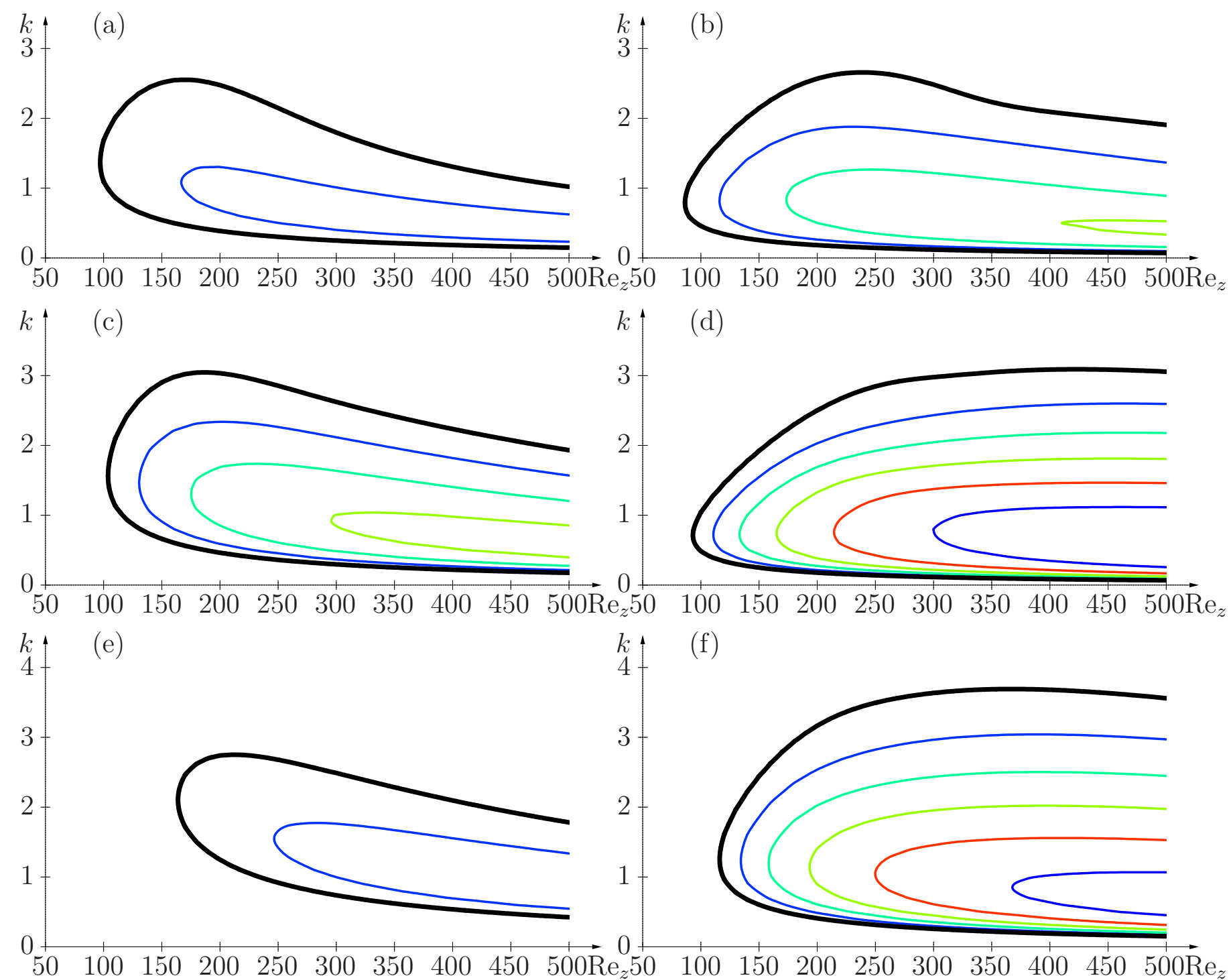

Figure 8. Relative axial mean flow-rate corrections for (a) $m=-1, \operatorname{Re}_{\Omega}=100$, (b) $m=-1, \operatorname{Re}_{\Omega}=200$, (c) $m=-2, \operatorname{Re}_{\Omega}=200$, (d) $m=-2, \operatorname{Re}_{\Omega}=500$, (e) $m=-3, \operatorname{Re}_{\Omega}=200$, (f) $m=-3, \operatorname{Re}_{\Omega}=500$. Thick black lines correspond to the marginal curve and thin coloured lines to levels $Q / Q_{\mathrm{bf}}=-0.05,-0.10,-0.15, \ldots$

maximum when $\operatorname{Re}_{z}$ is varied (see figure 1 ), both the flow-rate and angular momentum corrections continue to increase with the axial Reynolds number $\operatorname{Re}_{z}$ over the range investigated (see figures 8 and 9).

Finally, the frequencies of the linear and nonlinear travelling waves are compared in figure 10. For linear waves, the real part of the complex frequency $\omega_{r}^{\text {lin }}$ is derived from the linear dispersion relation (13) over the entire $\left(\mathrm{Re}_{z}, k\right)$-plane, and plotted with solid coloured lines in the panels of figure 10. Nonlinear waves exist within the regions of linear instability (delimited by thick black curves), and the dashed coloured lines indicate their associated frequency $\omega^{\mathrm{nl}}(18)$. These results show that the nonlinear waves always display a lower frequency than their linear counterparts. Due to the supercritical nature 

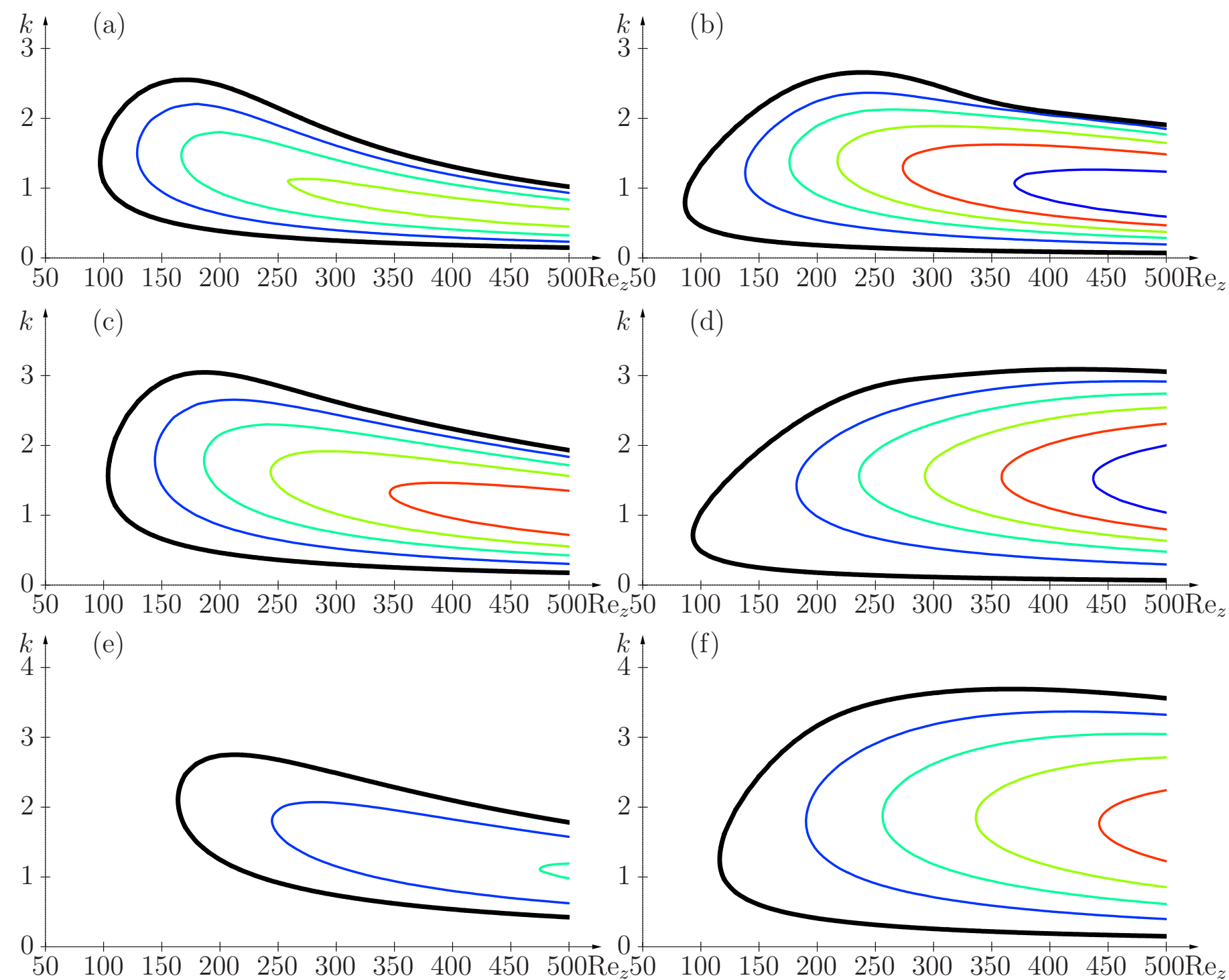

Figure 9. Relative mean angular momentum corrections for (a) $m=-1, \operatorname{Re}_{\Omega}=100$, (b) $m=-1, \operatorname{Re}_{\Omega}=200$, (c) $m=-2, \operatorname{Re}_{\Omega}=200$, (d) $m=-2, \operatorname{Re}_{\Omega}=500$, (e) $m=-3$, $\operatorname{Re}_{\Omega}=200$, (f) $m=-3, \operatorname{Re}_{\Omega}=500$. Thick black lines correspond to the marginal curve and thin coloured lines to levels $M / M_{\mathrm{bf}}=-0.01,-0.02,-0.03 \ldots$

of the present instabilities, linear and nonlinear frequencies converge to the same values along the curves of marginal stability. The departure between linear and nonlinear frequencies is seen to increase with $\mathrm{Re}_{z}$ and to be most significant towards the larger values of $k$.

\section{Conclusions}

In this paper, we have systematically investigated the fully developed dynamics prevailing in rotating Hagen-Poiseuille flow, for streamwise Reynolds numbers up to $\operatorname{Re}_{z}=500$ and azimuthal Reynolds numbers up to $\operatorname{Re}_{\Omega}=500$. 

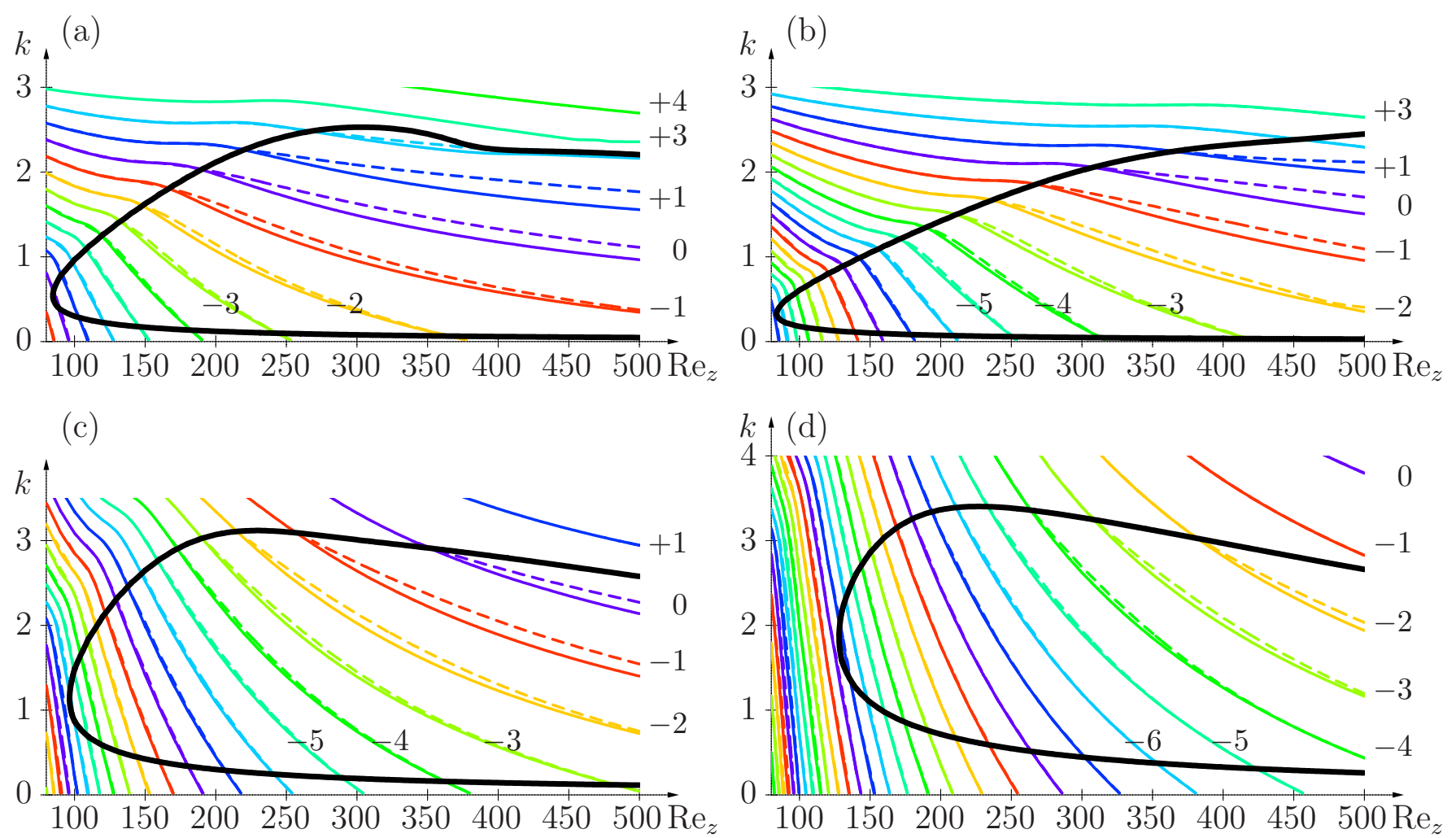

Figure 10. Frequencies of linear (solid coloured lines) and nonlinear (dashed lines) waves in the $\left(\operatorname{Re}_{z}, k\right)$-plane for (a) $m=-1, \operatorname{Re}_{\Omega}=300$, (b) $m=-1, \operatorname{Re}_{\Omega}=500$, (c) $m=-2, \operatorname{Re}_{\Omega}=300$, (d) $m=-3, \operatorname{Re}_{\Omega}=300$. The thick black lines indicate the curve of marginal stability.

In the small-amplitude regime, perturbations are governed by the Navier-Stokes equations linearized about the steady axisymmetric base flow and the relevant linear stability characteristics are derived by solving the associated eigenvalue problems for given values of the streamwise wavenumber $k$ and azimuthal modenumber $m$. The resulting dispersion relation has confirmed previously known results.

In unstable situations, small-amplitude perturbations may display exponential temporal growth and the resulting saturated fully developed regimes have been computed by direct numerical simulations of the complete Navier-Stokes equations. Over the entire control parameter space considered in this study, $\operatorname{Re}_{z} \leq 500$ and $\operatorname{Re}_{\Omega} \leq 500$, the flow fields have been found to evolve towards a nonlinear regime consisting of finite-amplitude travelling (i.e. spiralling) waves, characterised by constant values of energy levels, axial mean flow corrections and mean angular momentum corrections. The general effect of nonlinearities is to reduce the mean flow as well as the angular momentum. The strength of the axial mean-flow correction is directly related to the perturbation energy levels, and the highest levels occur for axial wavenumbers below those exhibiting strongest linear instability. The development of nonlinearities has always been found to result in a lower (or more negative) frequency of the nonlinear waves than of their linear counterparts. 
Some secondary instabilities have been observed at larger values of $\operatorname{Re}_{z}$ or $\operatorname{Re}_{\Omega}$ than those reported here. However, a complete study of secondary instability features is left for future investigation. Such secondary stability properties could be investigated by implementing a Floquet analysis similar to that used for the rotating-disk boundarylayer flow [19], and the resulting dynamics could be computed by simulations allowing for more degrees of freedom, e.g., replacing the single Fourier series in (14-17) by a double series expansion in both axial and azimuthal coordinates for the flow fields.

Since the RHPF is known to display absolute instabilities [5], it would also be interesting to consider domains of large streamwise extent as [15] to analyse the development of nonlinear global modes in terms of local nonlinear spiralling waves or for comparison with experimental observations [7]. Carrying out direct numerical simulations in axially extended domains would also allow a detailed investigation of inlet conditions and entry flow properties, which are known to have non-trivial effects for the non-rotating case [20]. Presumably, an approach similar to that presented in [21] could also shed light on how the global dynamics is influenced by the entry flow region.

\section{Acknowledgments}

The Indo-French Centre for the Promotion of Advanced Research/Centre francoindien pour la promotion de la recherche avancée (IFCPAR/CEFIPRA) is gratefully acknowledged for its financial support.

\section{References}

[1] Pedley T J 1968 J. Fluid Mech. 31 603-607

[2] Pedley T J 1969 J. Fluid Mech. 35 97-115

[3] Mackrodt P A 1976 J. Fluid Mech. 73 153-164

[4] Cotton F W and Salwen H 1981 J. Fluid Mech. 108 101-125

[5] Fernandez-Feria R and del Pino C 2002 Phys. Fluids 14 3087-3097

[6] Rusak Z and Wang S 2014 J. Fluid Mech. 759 321-359

[7] Miranda-Barea A, Fabrellas-Garca C, Parras1 L and del Pino C 2016 J. Fluid Mech. 793 316-334

[8] Imao S, Itoh M and Harada T 1996 Int. J. Heat and Fluid Flow 17 444-451

[9] Foster M R 2014 J. Fluid Mech. 749 113-144

[10] Toplosky N and Akylas T R 1988 J. Fluid Mech. 190 39-54

[11] Yang Z and Leibovich S 1991 J. Fluid Mech. 233 329-347

[12] Imao S, Itoh M, Yamada Y and Zhang Q 1992 Exp. Fluids 12 277-285

[13] Landman M J 1990 J. Fluid Mech. 221 289-310

[14] Barnes D R and Kerswell R R 2000 J. Fluid Mech. 417 103-126

[15] Sanmiguel-Rojas E and Fernandez-Feria R 2005 Phys. Fluids 17014104

[16] Dennis D J C, Seraudie C and Poole R J 2014 Phys. Fluids 26053602

[17] Pier B and Schmid P J 2017 J. Fluid Mech. 815 435-480

[18] Pier B 2015 Dynamique des écoulements ouverts : instabilités et transition, courbure et rotation Habilitation à diriger des recherches École centrale de Lyon \& Université de Lyon URL https://hal .archives-ouvertes.fr/tel-01107517

[19] Pier B 2007 J. Eng. Math. 57 237-251

[20] Sahu K C and Govindarajan R 2007 J. Fluids Eng. 129 1277-1280 
[21] Juniper M P and Pier B 2015 Eur. J. Mech. B-Fluids 49 426-437 